\title{
Convergence theorem of Pettis integrable multivalued pramart
}

\author{
M'Hamed El-Louh, Mohammed El Allali and Fatima Ezzaki \\ Laboratory of Modeling and Mathematical Structures, Faculty of Sciences and \\ Technologies, University Sidi Mohamed Ben Abdellah, Fez, Morocco
}

\begin{abstract}
Purpose - In this work, the authors are interested in the notion of vector valued and set valued Pettis integrable pramarts. The notion of pramart is more general than that of martingale. Every martingale is a pramart, but the converse is not generally true.

Design/methodology/approach - In this work, the authors present several properties and convergence theorems for Pettis integrable pramarts with convex weakly compact values in a separable Banach space.

Findings - The existence of the conditional expectation of Pettis integrable mutifunctions indexed by bounded stopping times is provided. The authors prove the almost sure convergence in Mosco and linear topologies of Pettis integrable pramarts with values in $(\mathrm{cwk}(\mathrm{E}))$ the family of convex weakly compact subsets of a separable Banach space.
\end{abstract}

Originality/value - The purpose of the present paper is to present new properties and various new convergence results for convex weakly compact valued Pettis integrable pramarts in Banach space.

Keywords Conditional expectation, Martingale, Pettis integrable pramart, Subpramart, Pettis integral,

Hausdorff distance, Stopping time, Mosco convergence

Paper type Research paper

\section{Introduction}

The set valued (alias multivalued) integration is useful in several areas of mathematics such as mathematical economics, image processing and analysis and theoretical statistics.

Various convergence results of set valued martingales and pramarts were studied in Bochner integration by several authors; see, Akhiat et al. [1], Akhiat et al. [2], Akhiat et al. [3], Castaing and Salvadori [4], Choukairi [5], Egghe [6], Ezzaki [7], Ezzaki and Tahri [8], Talagrand [9]. On the other hand, less of them is known in the case of Pettis integration. In the theory of integration in infinite-dimensional space, Pettis integrability is a more general concept than that of Bochner integrability. Examples of Pettis integrable functions, which are not Bochner integrable, are given in [10, Remark 5.2] and [11].

In this work, we are interested in the notion of vector valued and set valued Pettis integrable pramarts. The notion of pramart is more general than that of martingale. Every martingale is a pramart, but the converse is not generally true; see Egghe [6]. The purpose of the present paper is to present new properties and various new convergence results for convex weakly compact valued Pettis integrable pramarts in Banach space.

Our approach is based on a weak compactness result for Pettis integrable multifunctions and new results upon the existence of the conditional expectation of Pettis integrable multifunction developed in [12].

(C) M'Hamed El-Louh, Mohammed El Allali and Fatima Ezzaki. Published in the Arab Journal of Mathematical Sciences. Published by Emerald Publishing Limited. This article is published under the Creative Commons Attribution (CC BY 4.0) license. Anyone may reproduce, distribute, translate and create derivative works of this article (for both commercial and non-commercial purposes), subject to full attribution to the original publication and authors. The full terms of this license may be seen at http:// creativecommons.org/licences/by/4.0/legalcode

The authors wish to thank the referees for their helpful comments and remarks, which helped to improve this paper.
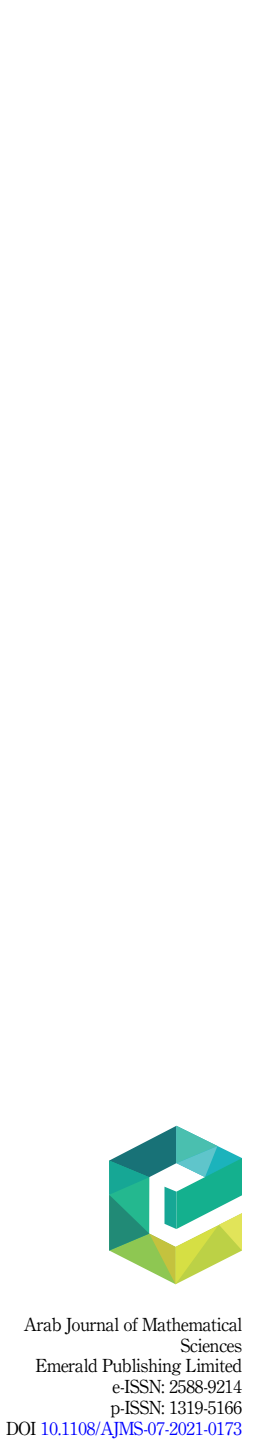
The paper is organized as follows. In Section 2, we give some preliminaries and needed results. In Section 3, we present the notion of Pettis integrability of convex weakly compact valued multifunction (alias set valued maps) indexed by bounded stopping times. The existence of the conditional expectation of aforementioned Pettis integrable multifunctions is also provided. In Section 4, we present some properties and almost sure convergence of vector valued Pettis integrable pramarts. By using the results stated in Section 3 and a weak compactness result for Pettis integrable multifunctions, we prove the almost sure convergence in Mosco and linear topologies of Pettis integrable pramarts with values in $(\operatorname{cwk}(\mathrm{E}))$ the family of convex weakly compact subsets of a separable Banach space $E$.

\section{Notations and preliminaries}

Throughout this paper, $(\Omega, \mathcal{A}, P)$ is a complete probability space, $\left(\mathcal{A}_{n}\right)_{n \geq 1}$ is an increasing sequence of sub $\sigma$-algebras of $\mathcal{A}$ such that $\mathcal{A}$ is the $\sigma$-algebra generated by $\cup_{n \geq 1} \mathcal{A}_{n}, E$ is a separable Banach space and $E^{*}$ is its topological dual. Let $D^{*}$ be a countable dense subset of $E^{*}$ with respect to the Mackey topology $\tau\left(E^{*}, E\right)$ and $B^{*}$ be the closed unit ball of $E^{*}$.

We denote by $c c(E)(\operatorname{resp} . c c b(E))(\operatorname{resp} . c w k(E))$ the set of nonempty convex and closed (resp. convex, closed and bounded) (resp. convex weakly compact) subsets of $E$. For $C \in 2^{E} \backslash \varnothing$, we denote by $c l A$ and $\overline{c o} A$ the closure and the closed convex hull of $A$ respectively and define $|C|=\sup \{\|x\|: x \in C\}$.

Let $C \in c c(E)$, the distance function and the support function associated to $C$ are defined respectively by

$$
\begin{aligned}
& d(x, C)=\inf \{\|x-y\|, y \in C\}, \quad x \in E, \\
& \delta^{*}\left(x^{*}, C\right)=\sup \left\{\left\langle x^{*}, y\right\rangle, y \in C\right\}, \quad x^{*} \in E^{*} .
\end{aligned}
$$

For any $A, B \in c c(E)$, the Hausdorff distance between $A$ and $B$ is defined by

$$
H(A, B)=\sup _{x^{*} \in B^{*}}\left|\delta^{*}\left(x^{*}, A\right)-\delta^{*}\left(x^{*}, B\right)\right| .
$$

$L_{E}^{1}\left(\right.$ resp. $\left.L^{1}\right)$ denotes the space of $\mathcal{A}$-measurable and Bochner integrable functions defined from $\Omega$ to $E$ (resp. the space of $\mathcal{A}$-measurable and integrable function defined from $\Omega$ to $\mathbb{R}$ ).

A set valued function $X: \Omega \rightarrow c c(E)$ is $\mathcal{A}$-measurable if for every open set $U \subset E$, the set

$$
X^{-} U=\{\omega \in \Omega: X(\omega) \cap U \neq \varnothing\}
$$

is in $\mathcal{A}$ see, [13].

A measurable set valued function is called a random set.

Let $\left(A_{n}\right)_{n \geq 1}$ be a sequence in $c c(E)$ and $A \in c c(E)$ we define

$$
s-l i A_{n}=\left\{x \in E:\left\|x_{n}-x\right\| \rightarrow_{n \rightarrow \infty} 0: x_{n} \in A_{n}, n \geq 1\right\},
$$

and

$$
w-l s A_{n}=\left\{x \in E: x=w-\lim _{j \rightarrow \infty} x_{j}: x_{j} \in A_{n_{j}}, j \geq 1\right\},
$$

where s (resp. $w$ ) is the strong (resp. weak) topology in $E$ and $\left(A_{n_{j}}\right)_{j \geq 1}$ is subsequence of $\left(A_{n}\right)_{n \geq 1}$.

We say that $\left(A_{n}\right)_{n \geq 1}$ is Mosco convergent to $A$, and we write $M-\lim A_{n}=A$ if $A=s-l i$ $A_{n}=w-l s A_{n}$. 
We say that $\left(A_{n}\right)_{n \geq 1}$ is convergent to $A$ in the linear topology, and we write $A=\tau_{L}-\lim$ $A_{n}$ if and only if the following properties are satisfied:

$-\lim _{n \rightarrow \infty} \delta^{*}\left(x^{*}, A_{n}\right)=\delta^{*}\left(x^{*}, A\right), \forall x^{*} \in E^{*}$

- $\lim _{n \rightarrow \infty} d\left(x, A_{n}\right)=d(x, A), \forall x \in E$.

For more properties of these topologies, see [14, Theorem 3.4]. On $c c(E)$, the linear topology is stronger than the Mosco topology; see, [14, Theorem 5.1].

Definition 21. A measurable function $f: \Omega \rightarrow E$ is said to be a selector of a random set $X$ if $f(\omega) \in X(\omega)$ for all $\omega \in \Omega$.

A measurable function $f: \Omega \rightarrow E$ is said to be scalarly integrable if $\left\langle x^{*}, f().\right\rangle \in L^{1}$ for each $x^{*} \in E^{*}$.

Definition 22. We say that fis Pettis integrable if it is scalarly integrable, and for each $A \in \mathcal{A}$, there exists $f_{A} \in E$ such that

$$
<x^{*}, f_{A}>=\int_{A}<x^{*}, f>d P, \quad \forall x^{*} \in E^{*} .
$$

$f_{A}$ is called the Pettis integral of fover $A$, and it is denoted by $\int_{A} f d P$.

We denote by $P_{E}^{1}(\mathcal{A})$ the space of all Pettis-integrable functions defined from $\Omega$ to $E . P_{E}^{1}(\mathcal{A})$ is endowed with the norm $\|\cdot\|_{P e}$ defined by

$$
\|f\|_{P e}=\sup _{x^{*} \in B^{*}} \int_{\Omega}\left|<x^{*}, f>\right| d P, \quad f \in P_{E}^{1}(\mathcal{A}) .
$$

An equivalent norm is given by $\|f\|_{P e}=\sup \left\{\left\|\int_{A} f d P\right\|: A \in \mathcal{A}\right\}$.

Definition 23. A subset $H \subset P_{E}^{1}$ is said to be uniformly Pettis integrable if $\sup _{f \in H}\|f\|_{P e}<$ $\infty$, and for each $\varepsilon>0$, there exists $\delta>0$ such that $\forall A \in \mathcal{A}$,

$$
P(A) \leq \delta \Rightarrow \sup _{x^{*} \in B^{*}} \int_{A}\left|<x^{*}, f>\right| d P \leq \varepsilon, \quad \forall f \in H .
$$

A random set $X$ with values in $c w k(E)$ is said to be scalarly integrable if $\forall x^{*} \in B^{*}, \delta^{*}\left(x^{*}, X\right)$ is integrable.

Definition 24. A random set $X: \Omega \rightarrow c w k(E)$ is said to be Pettis integrable in cwk $(E)$ if it is scalarly integrable, and for each $A \in \mathcal{A}$, there exists $C_{A} \in c w k(E)$ such that

$$
\delta^{*}\left(x^{*}, C_{A}\right)=\int_{A} \delta^{*}\left(x^{*}, X\right) d P, \quad \forall x^{*} \in E^{*} .
$$

$C_{A}$ is called the Pettis integral of $X$ over $A$, and it is denoted by $\int_{A} X d P$.

We denote by $P_{c w k(E)}^{1}(\mathcal{A})\left(\operatorname{resp} . P_{c c b(E)}^{1}(\mathcal{A})\right)$ the set of all Pettis-integrable random sets in $c w k(E)(\operatorname{resp} . c c b(E))$.

Let $S_{X}^{1}(\mathcal{A})\left(\right.$ resp. $S_{X}^{P e}(\mathcal{A})$ ) the set of all $\mathcal{A}$-measurable and Bochner integrable (resp. Pettis integrable) selectors of $X$,

$$
\begin{aligned}
& S_{X}^{1}(\mathcal{A})=\left\{f \in L_{E}^{1}(\mathcal{A}): f(\omega) \in X(\omega) \text { a.s. }\right\}, \\
& S_{X}^{P e}(\mathcal{A})=\left\{f \in P_{E}^{1}(\mathcal{A}): f(\omega) \in X(\omega) \text { a.s. }\right\} .
\end{aligned}
$$

integrable multivalued pramart 
$X$ is said to be Aumann-Pettis integrable if it is scalarly integrable and $S_{X}^{P e}$ is nonempty. The Aumann-Pettis integral of $X$ over $A \in \mathcal{A}$ is defined by $\left\{\int_{A} f d P, f \in S_{X}^{P e}(\mathcal{A})\right\}$.

Remark 1.

(1) Every Aumann-Pettis integrable random set X: $\Omega \rightarrow c c(E)$ is Pettis integrable in cc(E) (see, El Amri and Hess [15, Theorem 3.7]).

(2) A random set $X$ defined from $\Omega$ to cwk $(E)$ is Pettis integrable in cwk $(E)$ if and only if $\left\{\delta^{*}\left(x^{*}, X().\right), x^{*} \in B^{*}\right\}$ is uniformly integrable, see [15, Theorem 5.4]. In particular, every Pettis integrable random set $X$ in cwk $(E)$ is Aumann-Pettis integrable.

Before going further, we recall first the following results, which are one of the basic tools in the study of Pettis integrable multivalued random sets (resp. random variables).

Definition 25. A sequence $\left(X_{n}\right)_{n \geq 1}$ of random set with values in cc $(E)$ is said to be adapted to $\left(\mathcal{A}_{n}\right)_{n \geq 1}$ if for any $n \geq 1, X_{n}$ is $\mathcal{A}_{n}$-measurable.

Definition 26. Let $v: \mathcal{A} \rightarrow$ E be a measure. We say that $v$ is of $\sigma$-bounded variation if there exists a countable partition $\left(A_{n}\right)_{n \geq 1}$ of $\Omega$ in $\mathcal{A}$ such that the restriction $v_{\mid A_{n}}$ of $v$ to $A_{n}$ is a measure of bounded variation, for each $n \geq 1$.

Proposition 27. [1, Proposition 3.1]

Let $\left(A_{n}\right)_{n \in \mathbb{N}^{*}}$ be a sequence in cwk $(E)$. Assume that there exists $A_{\infty} \in c w k(E)$ and a sequence $\left(B_{n}\right)_{n \in \mathbb{N}^{*}}$ in cwk(E) such that:

(1) $\quad M-\lim _{n} A_{n}=A_{\infty}$.

Then

(2) $\lim _{n} H\left(A_{n}, B_{n}\right)=0$.

$$
M-\lim _{n} A_{n}=A_{\infty}=M-\lim _{n} B_{n}
$$

Proposition 28. [1, Proposition 3.2]

Let $D^{*}=\left\{e_{m}^{*}, m \in \mathbb{N}^{*}\right\}$ be a dense sequence in $B^{*}$ with respect to the Mackey topology $\tau\left(E^{*}, E\right)$. Let $\left(A_{n}\right)_{n \in \mathbb{N}^{*}}$ be a sequence in cwk $(E)$ and $A_{\infty}:=s-l i A_{n} \in \operatorname{cwk}(E)$.

Assume that

$$
\lim _{n} \delta^{*}\left(e_{m}^{*}, A_{n}\right)=\delta^{*}\left(e_{m}^{*}, A_{\infty}\right), \text { a.s. } \forall m \in \mathbb{N}^{*} .
$$

Then the following equality holds

$$
\lim _{n} d\left(x, A_{n}\right)=d\left(x, A_{\infty}\right) \text { a.s. } \forall x \in E .
$$

\section{Existence of the conditional expectation for Pettis integrable random sets indexed by stopping times}

Before giving the first main result of this section, let us recall the following definition.

A function $\tau: \Omega \rightarrow \mathbb{N}^{*} \cup\{+\infty\}$ is called a stopping time with respect to $\left(\mathcal{A}_{n}\right)_{n \in \mathbb{N}^{*}}$ if for each $n \in \mathbb{N}^{*},\{\tau=n\} \in \mathcal{A}_{n}$. The set of all bounded stopping times with respect to $\mathcal{A}_{n}$ is denoted by $T$.

For $\tau \in T$, we define the $\sigma$-algebra

$$
\mathcal{A}_{\tau}=\left\{A \in \mathcal{A}, A \cap\{\tau=n\} \in \mathcal{A}_{n}, \text { for each } n \geq 1\right\} .
$$

and 


$$
X_{\tau}=\sum_{n=\min \tau}^{n=\max } X_{n} \chi_{\{\tau=n\}} .
$$

Pettis integrable multivalued pramart

It is well known in the literature that the conditional expectation of Pettis-integrable random variables (resp. random sets) does not generally exist. Recently, several authors have studied the existence of this operator (see, Akhiat, Castaing et Ezzaki [1], Akhiat, El Harami et Ezzaki [16], El Allali et Ezzaki [12], El Harami et Ezzaki [17], Ezzaki et al. [18] and Ziat [19]).

Theorem 31. [1, Theorem 4.3]

Let $\mathcal{B}$ be a sub $\sigma$-algebra of $\mathcal{A}$ and $f$ be a Pettis integrable E-valued function such that $E^{\mathcal{B}}|f| \in[0, \infty]$.

Then there exists a unique a.s. $\mathcal{B}$-measurable, Pettis integrable E-valued function, denoted by $E^{\mathcal{B}} f$, which enjoys the following property:

For every $h \in L^{\infty}(\mathcal{B})$, one has

$$
\int_{\Omega} h E^{\mathcal{B}} f d P=\int_{\Omega} h f d P
$$

Definition 32. Let $\mathcal{B}$ be a sub- $\sigma$-algebra of $\mathcal{A}$ and $X$ be a Pettis integrable random set with values in cwk(E). We say that the conditional expectation of $X$ with respect to $\mathcal{B}$ exists if:

(1) $\forall f \in S_{X}^{P e}, E^{\mathcal{B}} f$ exists.

(2) There exists a $\mathcal{B}$-measurable random set $G$ such that

$$
\int_{A} G d P=\int_{A} X d P \quad \forall A \in \mathcal{B}
$$

Remark 2. If the conditional expectation of X exists, then it is unique a.s. In fact, Assume that there exists $G_{1}$ and $G_{2}$ which satisfies (32.1). Then for each $x^{*} \in E^{*}$

$$
\delta^{*}\left(x^{*}, G_{1}\right)=\delta^{*}\left(x^{*}, G_{2}\right) \text { a.e. }
$$

By [13, Lemma III.35], $G_{1}=G_{2}$ a.e.

Proposition 33. Let $X$ be an Aumann-Pettis integrable random set with values in cwk $(E)$ such that

(1) $\forall f \in S_{X}^{P e}, E^{\mathcal{B}} f$ exists,

(2) There exists a random set $G, \mathcal{B}$-measurable such that

$$
S_{G}^{P e}(\mathcal{B})=\overline{\left\{E^{\mathcal{B}} f / f \in S_{X}^{P e}\right\}^{\|}} \|_{P e} .
$$

Then $E^{\mathcal{B}} X$ exists and $E^{\mathcal{B}} X=G$.

Proof. Since $G$ is $\mathcal{B}$-measurable, it follows from El Amri and Hess [15, Theorem 3.9] that

$$
\begin{aligned}
& I_{A}^{(\mathcal{B})}(G)=I_{A}(G) . \forall f \in S_{X}^{P e}, \\
& <x^{*}, \int_{A} E^{\mathcal{B}} f d P>=\int_{A}<x^{*}, f>d P \leq \delta^{*}\left(x^{*}, \int_{A} X d P\right) \forall x^{*} \in E^{*}, \forall A \in \mathcal{B} .
\end{aligned}
$$


Then

$$
\delta^{*}\left(x^{*}, I_{A}(G)\right) \leq \delta^{*}\left(x^{*}, \int_{A} X d P\right)
$$

Hence

$$
\int_{A} G d P=I_{A}(G) \subset \int_{A} X d P
$$

Conversely, let $f \in I_{A}(X)$

$$
<x^{*}, \int_{A} f d P>=<x^{*}, \int_{A} E^{\mathcal{B}} f d P>\leq \delta^{*}\left(x^{*}, I_{A}(G)\right) \forall x^{*} \in E^{*}, \forall A \in \mathcal{B} .
$$

Then

$$
\delta^{*}\left(x^{*}, I_{A}(X)\right) \leq \delta^{*}\left(x^{*}, I_{A}(G)\right), \forall x^{*} \in E^{*} .
$$

So,

$$
\int_{A} X d P \subset \int_{A} G d P
$$

Before giving the first main result of this section, let us present useful theorem due to El Allali and Ezzaki [20], which will be used in the proof of theorem 36.

Theorem 34. Let $\mathcal{B}$ be a sub $\sigma$-algebra of $\mathcal{A}$ and $X$ be an Aumann-Pettis integrable random set with values in cwk $(E)$ such that there exists a countable partition $\left(B_{k}\right)_{k \geq 1}$ of $\Omega$ in $\mathcal{B}$ such that

$$
\int_{B_{k}}|X| d P<\infty, \forall k \geq 1
$$

Then.

(1) $E^{\mathcal{B}} X$ exists, and it is with values in cwk(E).

(2) $E^{\mathcal{B}} X=\overline{c o}\left\{E^{\mathcal{B}} f_{n}, n \geq 1\right\}$ a.s.,

(3) $\forall A \in \mathcal{B}, \int_{A} X d P=\int_{A} E^{\mathcal{B}} X d P$,

(4) $S_{E^{\mathcal{B} X}}^{P e}=\overline{\left\{E^{\mathcal{B}} f / f \in S_{X}^{P e}\right\}^{\|}} \|_{\|_{e}}$.

Proof. The proof of (1) and (2) of the theorem where proved in [20, theorem 3.9], we will provide some details of the proof for the sake of completeness.

Let $M=\left\{E^{\mathcal{B}} f, f \in S_{X}^{P_{e}}\right\}$. It is clear that $M \subset \mathcal{P}_{E}(\mathcal{B})$, convex, nonempty and decomposable with respect to $\mathcal{B}$. Let $D=\left\{x_{n}^{*}, n \geq 1\right\}$ be a subset of $B^{*}$ such that $x_{n}^{*}=\frac{y_{n}^{*}}{\left\|y_{n}^{*}\right\|}$ with $\left(y_{n}^{*}\right)_{n \geq 1}$ is a dense sequence in $E^{*}$ with respect to the Mackey topology $\tau\left(E^{*}, E\right)$.

Let $\left(E^{\mathcal{B}} f_{i}\right)_{i \geq 1}$ be a sequence in $M$. We shall prove that the random set

$$
H(w)=\overline{c o}\left\{E^{\mathcal{B}} f_{i}(w), i \geq 1\right\}, \quad w \in \Omega
$$

is convex and weakly compact a.s. 
Let $n \geq 1, X_{n}=X_{\mid B_{n}}, H_{n}=H_{\mid B_{n}}$ and $\mathcal{B}_{n}=\left\{A \cap B_{n}, A \in \mathcal{B}\right\}$. It follows from [21, Theorem 3.2] that $S_{X_{n}}^{1}$ is convex and weakly compact, then $\left\{E^{\mathcal{B}_{n}} f, f \in S_{X_{n}}^{1}\right\}$ is convex and weakly compact. On the other hand $S_{H_{n}}^{1}\left(\mathcal{B}_{n}\right) \subset\left\{E^{\mathcal{B}_{n}} f, f \in S_{X_{n}}^{1}\right\}$. Indeed, let

$$
G(w)=\overline{\left\{\left(E^{\mathcal{B}} f_{i}\right)_{\mid B_{n}}(w), i \geq 1\right\}}=\overline{\left\{E^{\mathcal{B}_{n}} f_{i \mid B_{n}}(w), i \geq 1\right\}}, \quad w \in B_{n} .
$$

$G$ is $\mathcal{B}_{n}$ measurable and $\forall i \geq 1, E^{\mathcal{B}_{n}} f_{i \mid B_{n}} \in\left\{E^{\mathcal{B}_{n}} f, f \in S_{X_{n}}^{1}\right\}$, on the other hand $\left\{E^{\mathcal{B}_{n}} f, f \in S_{X_{n}}^{1}\right\}$ is closed and decomposable with respect to $\mathcal{B}_{n}$, then it follows from [22, Lemma 1.3] that $S_{G}^{1}\left(\mathcal{B}_{n}\right) \subset\left\{E^{\mathcal{B}_{n}} f, f \in S_{X_{n}}^{1}\right\}$, then $S_{H_{n}}^{1}\left(\mathcal{B}_{n}\right)=\overline{c o} S_{G}^{1}\left(\mathcal{B}_{n}\right) \subset\left\{E^{\mathcal{B}_{n}} f, f \in S_{X_{n}}^{1}\right\}$.

Hence $S_{H_{n}}^{1}\left(\mathcal{B}_{n}\right)$ is convex and weakly compact since it is closed. So it follows from [21, Theorem 3.2] that $\forall n \geq 1, H_{n}$ is convex and weakly compact a.s., then $\mathrm{H}$ is countably supported a.s. with respect to $D$. Thus by $[12$, Theorem 35$]$ there exist a $\mathcal{B}$-measurable random set $L$ and a sequence $\left(f_{n}\right)_{n \geq 1} \subset S_{X}^{P_{e}}$ such that $L(w)=\overline{c o}\left\{E^{\mathcal{B}} f_{n}(w), n \geq 1\right\}$ for each $w \in \Omega$ and $S_{L}^{P_{e}}(\mathcal{B})=\bar{M}^{\|\cdot\|_{P_{e}}}$.

Let $A \in \mathcal{B}$ such that $P(A)=0$ and $L(w) \in c w k(E), \forall w \in \Omega \backslash A$. Let.

$E^{\mathcal{B}} X(w)= \begin{cases}L(w) & , w \in \Omega \backslash A . \\ \{0\} & , w \in A .\end{cases}$

$E^{\mathcal{B}} X$ is a $\mathcal{B}$-measurable random set with values in $c w k(E)$ and $S_{E^{\mathcal{B}} X}^{P_{e}}(\mathcal{B})=S_{L}^{P_{e}}(\mathcal{B})=\bar{M}^{\|\cdot\|_{P_{e}}}$. Moreover, $E^{\mathcal{B}} X(w)=\overline{c o}\left\{E^{\mathcal{B}} f_{n}(w), n \geq 1\right\}$ a.s.

(3) Since $E^{\mathcal{B}} X$ exists by (1) it is clear from the definition of conditional expectation (see definition 32)

$$
\forall A \in \mathcal{B}, \int_{A} X d P=\int_{A} E^{\mathcal{B}} X d P
$$

(4) It follows from proposition 33 with $G=E^{\mathcal{B}} X$.

Proposition 35. Let $X$ be an Aumann-Pettis integrable random set with values in cwk $(E)$ such that there exists a countable partition $\left(B_{n}\right)_{n \geq 1}$ of $\Omega$ in $\mathcal{B}$ that satisfies

$$
\int_{B_{n}}|X| d P<\infty, \forall n \geq 1
$$

Then, $\left\{E^{\mathcal{B}} f / f \in S_{X}^{P e}\right\}$ is closed in $P_{E}^{1}$.

Proof. From (35.1), every selection of $X \cdot \chi_{B_{n}}$ is Bochner integrable then $S_{X \cdot \chi_{B_{n}}}^{1}$ $(\mathcal{B})=S_{X \cdot \chi_{B n}}^{P e}(\mathcal{B}) \forall n \geq 1$

Since $X$ is with values in $c w k(E)$, by [21, Theorem 3.2] $S_{X \cdot \chi_{B_{n}}}^{P e}$ is weakly compact for each $n \geq 1$. So

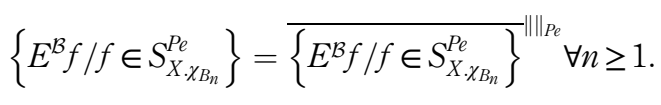

Now, let $g \in P_{E}^{1}$ such that there exists $f_{n} \in S_{X}^{P e}$ such that

$$
E^{\mathcal{B}} f_{n} \rightarrow g \text { in } P_{E}^{1}
$$


For each $\left.n \geq 1, g \cdot \chi_{B_{n}} \in \overline{\left\{E^{\mathcal{B}} f / f \in S_{X \cdot \chi_{B_{n}}}^{P e}\right.}\right\}^{\|\|_{P e}}=\left\{E^{\mathcal{B}} f / f \in S_{X \cdot \chi_{B_{n}}}^{P e}\right\}$. Then for each $n \geq 1$, there exists $h_{n} \in S_{X \cdot \chi_{B n}}^{P e}$ such that $g_{n}=E^{\mathcal{B}} h_{n}$.

Set $h=\sum_{n \geq 1} h_{n} \cdot \chi_{B_{n}}$. It is clear that $h$ is a selector of $X$.

Since $X$ is Pettis integrable with values in $c w k(E), h$ is Pettis integrable. On the other hand, $g=E^{\mathcal{B}} h$.

Hence $\left\{E^{\mathcal{B}} f / f \in S_{X}^{P e}\right\}$ is closed in $P_{E}^{1}$.

In the following theorem, we prove the existence of the conditional expectation of Pettis integrable multifunctions indexed by bounded stopping times, which will allow us to well define the notion of vector valued and set valued pramart in the Pettis integration.

Theorem 36. Let $Y$ be a positive random variable such that $E^{\mathcal{A}_{1}} Y<\infty$. Let $\left(X_{n}\right)_{n \geq 1}$ be a sequence of Pettis integrable random sets with values in a family $\mathrm{cwk}(E)$ such that

$\left|X_{n}\right| \leq Y \forall n \geq 1$. Let $\sigma$ and $\tau \in T$ such that $\tau \geq \sigma$. Then $X_{\tau}$ is a Pettis integrable random set with values in cwk $(E)$ and the conditional expectation of $X_{\tau}$ with respect to $\mathcal{A}_{\sigma}$ exists, and satisfies the following properties;

(1) $S_{E^{\mathcal{A}_{\sigma}} X_{\tau}}^{P e}=\left\{E^{\mathcal{A}_{\sigma}} f / f \in S_{X_{\tau}}^{P e}\right\}$

(2) $\forall A \in \mathcal{A}_{\sigma}, \int_{A} X_{\tau} d P=\int_{A} E^{\mathcal{A} \sigma} X_{\tau} d P$.

Proof. For $\tau \in T$,

$$
X_{\tau}=\sum_{k=\min \tau}^{k=\max \tau} X_{k} \chi_{[\tau=k]} .
$$

$\forall k \geq 1, X_{k}$ is a Pettis integrable random set, then $X_{\tau}$ is a Pettis integrable random set in $P_{c w k(E)}^{1}(\mathcal{A})$.

In fact, $X_{\tau}$ is $\mathcal{A}$-measurable, scalarly integrable and $\forall x^{*} \in E^{*}, \forall A \in \mathcal{A}$

$$
\begin{aligned}
\int_{A} \delta^{*}\left(x^{*}, X_{\tau}\right) d P & =\int_{A} \delta^{*}\left(x^{*}, \sum_{k=\min \tau}^{k=\max \tau} X_{k} \chi_{[\tau=k]}\right) d P=\sum_{k=\min \tau}^{k=\max \tau} \int_{A} \delta^{*}\left(x^{*}, X_{k} \chi_{[\tau=k]}\right) d P \\
& =\sum_{k=\min \tau}^{k=\max \tau} \delta^{*}\left(x^{*}, \int_{A} X_{k} \chi_{[\tau=k]} d P\right)=\delta^{*}\left(x^{*}, \sum_{k=\min \tau}^{k=\max \tau} \int_{A} X_{k} \chi_{[\tau=k]} d P\right) \\
& =\delta^{*}\left(x^{*}, \int_{A}^{k=\max \tau} \sum_{k=\min \tau} X_{k} \chi_{[\tau=k]} d P\right)=\delta^{*}\left(x^{*}, \int_{A} X_{\tau} d P\right)
\end{aligned}
$$

Then $X_{\tau}$ is a Pettis integrable random set with values in $c w k(E)$.

Now, we prove that the conditional expectation of $X_{\tau}$ with respect to $\mathcal{A}_{\sigma}$ exists.

Since $X_{\tau}=\sum_{k=\min \tau}^{k=\max \tau} X_{k} \chi_{[\tau=k}, \forall \omega \in \Omega,\left|X_{\tau}(\omega)\right| \leq Y(\omega)$.

The fact that $E^{\mathcal{A}_{1}} Y<\infty$, there exists a partition of $\Omega$ in $\mathcal{A}_{1}$ such that 


$$
\begin{aligned}
\int_{B_{k}}\left|X_{\tau}\right| d P & =\int_{B_{k}}\left|\sum_{j=\min \tau}^{j=\max \tau} X_{j} \chi_{[\tau=j]}\right| d P \\
& \leq \int_{B_{k}} \sum_{j=\min \tau}^{j=\max \tau}\left|X_{j} \chi_{[\tau=]}\right| d P \\
& \leq \sum_{j=\min \tau}^{j=\max \tau} \int_{B_{k}} Y d P<\infty \quad \forall k \geq 1 .
\end{aligned}
$$

Pettis integrable multivalued

Since $\mathcal{A}_{1} \subset \mathcal{A}_{k} \forall k \geq 1$ and

$$
X_{\tau}=\sum_{j=\min \tau}^{j=\max \tau} X_{j} \chi_{[\tau=]]} .
$$

Thus by theorem $34, E^{\mathcal{A}_{k}} X_{j} \chi_{[\tau=j]}$ exists $\forall k \geq 1$.

The fact that,

$$
E^{\mathcal{A} \sigma} X_{\tau}=\sum_{k=\min \sigma}^{k=\max \sigma} \chi_{[\sigma=k]} \sum_{j=\min \tau}^{j=\max \tau} E^{\mathcal{A}_{k}} X_{j} \chi_{[\tau=j]}
$$

Then $E^{\mathcal{A}_{\sigma}} X_{\tau}$ exists and from proposition 33,

$$
S_{E^{\mathcal{A}_{\sigma}} X_{\tau}}^{P e}=\left\{E^{\mathcal{A}_{\sigma}} f / f \in S_{X_{\tau}}^{P e}\right\}
$$

Now, we will show that $\forall A \in \mathcal{A}_{\sigma}, \int_{A} X_{\tau} d P=\int_{A} E^{\mathcal{A} \sigma} X_{\tau} d P$.

Let $A \in \mathcal{A}_{\sigma}$ and set $B_{j}=A \cap[\sigma=j]$

$$
\begin{aligned}
\delta^{*}\left(x^{*}, \int_{B_{j}} E^{\mathcal{A}_{\sigma}} X_{\tau}\right) d P=\delta^{*}\left(x^{*}, \int_{B_{j}} E^{\mathcal{A}_{j}} X_{\tau} d P\right) & =\delta^{*}\left(x^{*}, \int_{B_{j}} E^{\mathcal{A}_{j}} \sum_{k \geq j}^{\max \tau} X_{k} \chi_{[\tau=k]} d P\right) \\
& =\int_{B_{j}} \delta^{*}\left(x^{*}, E^{\mathcal{A}_{j}} \sum_{k \geq j}^{\max \tau} X_{k} \chi_{[\tau=k]}\right) d P \\
& =\int_{B_{j}} E^{\mathcal{A}_{j}} \delta^{*}\left(x^{*}, \sum_{k \geq j}^{\max \tau} X_{k} \chi_{[\tau=k]}\right) d P \\
& =\int_{B_{j}} \delta^{*}\left(x^{*}, X_{\tau}\right) d P
\end{aligned}
$$

$A=\cup_{j=\min \sigma}^{j=\max \sigma} B_{j}$ so, 
AJMS

$$
\begin{aligned}
\delta^{*}\left(x^{*}, \int_{A} E^{\mathcal{A}_{\sigma}} X_{\tau}\right) d P & =\sum_{j=\min \sigma}^{j=\max \sigma} \int_{B_{j}} E^{\mathcal{A}_{j}} \delta^{*}\left(x^{*}, X_{\tau}\right) d P \\
& =\sum_{j=\max \sigma}^{j=\min \sigma} \int_{B_{j}} \delta^{*}\left(x^{*}, X_{\tau}\right) d P \\
& =\int_{A} \delta^{*}\left(x^{*}, X_{\tau}\right) d P \\
& =\delta^{*}\left(x^{*}, \int_{A} X_{\tau} d P\right) \forall A \in \mathcal{A}_{\sigma}
\end{aligned}
$$

Then

$$
\int_{A} E^{\mathcal{A}_{\sigma}} X_{\tau} d P=\int_{A} X_{\tau} d P, \forall A \in \mathcal{A}_{\sigma}
$$

Now, let us introduce some needed definitions and notions of pramarts (resp. subpramarts) in $P_{E}^{1}(\mathcal{A})$ and in $P_{c w k(E)}^{1}(\mathcal{A})$.

Definition 37. A Pettis integrable adapted sequence $\left(X_{n}, \mathcal{A}_{n}\right)_{n \geq 1}$ with values in $c c(E)$ is said to be a martingale if for each $k \geq n \geq 1, E^{\mathcal{A}_{n}} X_{k}$ exists and $X_{n}=E^{\mathcal{A}_{n}} X_{k}$ a.s.

Definition 38. Let $\left(X_{n}, \mathcal{A}_{n}\right)_{n \geq 1}$ be an adapted sequence in $P_{E}^{1}(\mathcal{A})$. Assume that for every $\sigma, \tau$ $\in T, \tau \geq \sigma$ the conditional expectation $E^{\mathcal{A}_{\sigma}} X_{\tau} \in P_{E}^{1}(\mathcal{A})$ exists.

We say that $\left(X_{n}, \mathcal{A}_{n}\right)_{n \geq 1}$ is a pramart if for every $\varepsilon>0$, there is $\sigma_{\varepsilon} \in T$ such that,

$\forall \sigma, \tau \in T, \quad \tau \geq \sigma \geq \sigma_{\varepsilon} \Rightarrow P\left(\left\|X_{\sigma}-E^{\mathcal{A}_{\sigma}} X_{\tau}\right\|>\varepsilon\right) \leq \varepsilon$.

Definition 39. Let $\left(X_{n}, \mathcal{A}_{n}\right)_{n \geq 1}$ be an adapted sequence in $L_{\mathbb{R}}^{1}(\mathcal{A})$. We say that $\left(X_{n}, \mathcal{A}_{n}\right)_{n \geq 1}$ is a subpramart if for every $\varepsilon>0$, there is $\sigma_{\varepsilon} \in T$ such that,

$\forall \sigma, \tau \in T, \quad \tau \geq \sigma \geq \sigma_{\varepsilon} \Rightarrow P\left(\left(X_{\sigma}-E^{\mathcal{A}_{\sigma}} X_{\tau}\right)^{+}>\varepsilon\right) \leq \varepsilon$.

Definition 310. Let $\left(X_{n}, \mathcal{A}_{n}\right)_{n \geq 1}$ be an adapted sequence in $P_{c w k(E)}^{1}(\mathcal{A})$. Assume that for every $\sigma, \tau \in T, \tau \geq \sigma$ the conditional expectation $E^{\mathcal{A}_{\sigma}} X_{\tau} \in P_{\text {cwk }(E)}^{1}(\mathcal{A})$ exists.

We say that $\left(X_{n}, \mathcal{A}_{n}\right)_{n \in \mathbb{N}}$ is a pramart if for every $\varepsilon>0$, there is $\sigma_{\varepsilon} \in T$ such that,

$\forall \sigma, \tau \in T, \quad \tau \geq \sigma \geq \sigma_{\varepsilon} \Rightarrow P\left(H\left(X_{\sigma}, E^{\mathcal{A}_{\sigma}} X_{\tau}\right)>\varepsilon\right) \leq \varepsilon$.

Proposition 311. Let $\mathcal{B}$ be a sub $\sigma$-algebra of $\mathcal{A}$ and $X: \Omega \rightarrow$ cwk $(E)$ be a Pettis integrable random set such that $E^{\mathcal{B}}|X|<\infty$ and $f \in P_{E}^{1}(\mathcal{A})$, then $f \in S_{E^{\mathcal{B}} X}^{P e}$ if and only if $\int_{A} f(\omega) d P \in \int_{A} X(\omega) d P$ for all $A \in \mathcal{A}$.

Proof. Let $X \in P_{c w k(E)}^{1}(\mathcal{A})$ such that $E^{\mathcal{B}}|X|<\infty$ then by theorem $34, E^{\mathcal{B}} X$ exists and

$$
S_{E^{\mathcal{B} X}}^{P e}=\left\{E^{\mathcal{B}} f: f \in S_{X}^{P e}\right\} .
$$

Let $f \in S_{E^{\mathcal{B} X}}^{P e}$ and $\left(x_{m}^{*}\right)_{m \geq 1}$ be a dense sequence in $E^{*}$ with respect to Mackey topology $\tau\left(E^{*}, E\right)$ 
and $A \in \mathcal{A}$.

$$
\int_{A}<x_{m}^{*}, f>d P \leq \int_{A} \delta^{*}\left(x_{m}^{*}, E^{\mathcal{B}} X\right) d P .
$$

Pettis integrable multivalued pramart

Then

$$
<x_{m}^{*}, \int_{A} f d P>\leq \delta^{*}\left(x_{m}^{*}, \int_{A} E^{\mathcal{B}} X d P\right) .
$$

Hence

$$
\int_{A} f d P \in \int_{A} X d P \text { for all } A \in \mathcal{A} .
$$

Let show the converse implication, from [2, Lemma 3.4] $\forall x^{*} \in E^{*}$,

$$
E^{\mathcal{B}}\left(\delta^{*}\left(x^{*}, X\right)\right)=\delta^{*}\left(x^{*}, E^{\mathcal{B}} X\right) \text { a.s. }
$$

For every $m \geq 1$

$$
<x_{m}^{*}, \int_{A} f d P>\leq \delta^{*}\left(x_{m}^{*}, \int_{A} X d P\right)
$$

So,

$$
\begin{gathered}
\int_{A}<x_{m}^{*}, f>d P \leq \int_{A} \delta^{*}\left(x_{m}^{*}, X\right) d P=\int_{A} E^{\mathcal{B}} \delta^{*}\left(x_{m}^{*}, X\right) d P \text { for all } A \in \mathcal{A} . \\
\Rightarrow<x_{m}^{*}, f(\omega)>\leq \delta^{*}\left(x_{m}^{*}, E^{\mathcal{B}} X(\omega)\right) \text { a.s. }
\end{gathered}
$$

So, there exists a negligible $N=\cup_{m} N_{m}$ such that $\forall \omega \in \Omega \backslash N, \forall x_{m}^{*} \in D^{*}$,

$$
<x_{m}^{*}, f(\omega)>\leq \delta^{*}\left(x_{m}^{*}, E^{\mathcal{B}} X(\omega)\right) .
$$

By [13, Lemma III.34], $f(\omega) \in E^{\mathcal{B}} X(\omega)$ Then $f \in S_{E^{\mathcal{B} X}}^{P e}$.

\section{Convergence theorem of Pettis integrable vector valued and set valued pramart}

Before presenting our convergence results, we recall the following definition and properties of multimeasure, which will be used in the proof of theorem 410 at the end of this section.

Definition 41. let $E$ be any Banach space. A multimeasure is a map $M: \mathcal{A} \rightarrow 2^{E} \backslash \varnothing$ such that $M(\varnothing)=\{0\}$ and for every $\left(A_{n}\right)_{n \geq 1}$ in $\mathcal{A}$ pairwise disjoint we have

Given a map $M: \mathcal{A} \rightarrow c w k(E)$ :

$$
M\left(\cup_{n} A_{n}\right)=\sum_{n} M\left(A_{n}\right)
$$

(1) By Costé [23], $M$ is a multimeasure if and only if for every $x^{*} \in E^{*}, \delta^{*}\left(x^{*}, M().\right)$ is a finite scalar measure. 
(2) The variation of $M$ is denoted by $|M|$ and defined by

$$
|M(A)|=\sup \sum_{j}\left|M\left(A_{j}\right)\right|, A \in \mathcal{A} .
$$

The sup is taken on all the finite partitions of $A$ in $\mathcal{A}$.

Now, we present the following Lemmas, which will be used in the proof of our main result in this section.

Lemma 42. Let $\left(C_{n}\right)_{n \geq 1}$ be a sequence of Pettis integrable random sets with values in cwk $(E)$ satisfying the following conditions:

(1) There exists a Pettis integrable random set $L$ with values in cwk $(E)$ such that $C_{n}(.) \subset L($. $\forall n \geq 1$.

(2) $\lim _{n} \delta^{*}\left(x^{*}, C_{n}(\omega)\right)$ exists $\forall \omega \in \Omega, \forall x^{*} \in D^{*}$.

Then there exists a Pettis integrable random set $C$ with values in $\mathrm{cwk}(E)$ such that

$$
\lim _{n} \delta^{*}\left(x^{*}, C_{n}(\omega)\right)=\delta^{*}\left(x^{*}, C(\omega)\right), \quad \forall x^{*} \in E^{*}, \quad \forall \omega \in \Omega .
$$

Proof. By assumption, $C_{n}(\omega) \subset L(\omega), \forall \omega \in \Omega, \forall n \geq 1$, then $\left(\delta^{*}\left(., C_{n}(\omega)\right)\right)_{n \geq 1}$ is an equicontinuous sequence with respect to the Mackey topology $\tau\left(E^{*}, E\right)$.

Let $\omega \in \Omega$, and set $r()=.\lim _{n} \delta^{*}\left(\right.$., $\left.C_{n}(\omega)\right)$. So $r($.$) is continuous with respect to the Mackey$ topology. Since $r($.) is positively homogeneous and $r(0)=0$, then there exists $C(\omega) \in c c(E)$ such that

$$
\lim _{n} \delta^{*}\left(x^{*}, C_{n}(\omega)\right)=\delta^{*}\left(x^{*}, C(\omega)\right), \quad \forall x^{*} \in E^{*}, \forall \omega \in \Omega
$$

So,

$$
\delta^{*}\left(x^{*}, C(\omega)\right) \leq \delta^{*}\left(x^{*}, L(\omega)\right), \quad \forall x^{*} \in E^{*}, \forall \omega \in \Omega,
$$

then $C(\omega) \in c w k(E), \forall \omega \in \Omega$.

By [24, Lemma 5.2], $C($.) is measurable.

Let us now prove that $C($.$) is Pettis integrable in c w k(E)$. By [15, Theorem 5.4] it is sufficient to prove that $\left\{\delta^{*}\left(x^{*}, C().\right) x^{*} \in B^{*}\right\}$ is uniformly integrable.

For every $x^{*} \in B^{*}$

$$
\begin{gathered}
\delta^{*}\left(x^{*}, C(\omega)\right)^{+} \leq \delta^{*}\left(x^{*}, L(\omega)\right)^{+}, \\
\delta^{*}\left(x^{*}, C(\omega)\right)^{-} \leq \delta^{*}\left(-x^{*}, L(\omega)\right)^{+} .
\end{gathered}
$$

Then

$$
\sup _{x^{*} \in B^{*}} \int_{\Omega}\left|\delta^{*}\left(x^{*}, C(\omega)\right)\right| d P \leq 2 \sup _{x^{*} \in B^{*}} \int_{\Omega}\left|\delta^{*}\left(x^{*}, L(\omega)\right)\right| d P<\infty .
$$

For every $\varepsilon>0, \exists \delta>0$ such that $\forall A \in \mathcal{A}, P(A)<\delta \Rightarrow$

$$
\sup _{x^{*} \in B^{*}} \int_{A}\left|\delta^{*}\left(x^{*}, L(\omega)\right)\right| d P<\varepsilon .
$$


Then

$$
\sup _{x^{*} \in B^{*}} \int_{A}\left|\delta^{*}\left(x^{*}, C(\omega)\right)\right| d P \leq 2 \sup _{x^{*} \in B^{*}} \int_{A}\left|\delta^{*}\left(x^{*}, L(\omega)\right)\right| d P<2 \varepsilon .
$$

So, $\left\{\delta^{*}\left(x^{*}, C().\right), x^{*} \in B^{*}\right\}$ is uniformly integrable.

The first application of lemma 42 in $P_{E}^{1}$ is the following lemma, which proofs the convergence in Pettis norm of Pettis integrable pramart. Since the notion of pramart is more general than that of martingale, the result proved here generalizes the convergence in Pettis norm of martingales proved in [25, lemma 1.4] and [18, lemma 2].

Lemma 43. Assume that $E^{*}$ is separable. Let $\left(f_{n}, \mathcal{A}_{n}\right)_{n \geq 1}$ be a pramart in $P_{E}^{1}(\mathcal{A})$ such that the following conditions are satisfied:

(1) There is a Pettis integrable random set $L: \Omega \rightarrow c w k(E)$ such that $f_{n}(\omega) \in L(\omega)$ for all $n \geq 1$ and all $\omega \in \Omega$.

(2) For each $n \geq 1, E^{\mathcal{A}_{n}}|L|<\infty$.

Then there exists $f \in P_{E}^{1}(\mathcal{A})$ such that.

$\left\|f_{n}-f\right\|_{P e} \rightarrow 0$.

Proof. By (2) $\forall n, \forall k \geq n E^{\mathcal{A}_{n}} f_{k}$ exists.

Since $f_{n}(\omega) \in L(\omega)$ then

$$
\sup _{n} \sup _{x^{*} \in B^{*}} \int_{\Omega}\left|<x^{*}, f_{n}(\omega)>\right| d P \leq 2 \sup _{x^{*} \in B^{*}} \int_{\Omega}\left|\delta^{*}\left(x^{*}, L(\omega)\right)\right| d P<\infty .
$$

Then for each $x^{*} \in B^{*},\left(<x^{*}, f_{n}(.)>, \mathcal{A}_{n}\right)_{n \geq 1}$ is a $L^{1}$-bounded pramart. Then from Theorem 1.1 in $\left.[26]\left(<x^{*}, f_{n}().\right\rangle\right)_{n \geq 1}$ converges a.s. to $\varphi_{x^{*}}($.$) .$

So by lemma 42 there exists $f($.$) such that for each x^{*} \in E^{*}$,

$$
\varphi_{x^{*}}(.)=<x^{*}, f(.)>
$$

We conclude that

$$
\lim _{n \rightarrow \infty}<x^{*}, f_{n}(\omega)>=<x^{*}, f(\omega)>\text {, a.s. } \forall x^{*} \in E^{*} .
$$

Since $L$ is Pettis integrable with values in $\operatorname{cwk}(E)$ then $\left\{\delta^{*}\left(x^{*}, L\right), x^{*} \in B^{*}\right\}$ is uniformly integrable, so it is not hard to see that $\left.\forall x^{*} \in B^{*},\left\{<x^{*}, f_{n}().\right\rangle, n \geq 1\right\}$ is uniformly integrable pramart. Then

$$
\forall x^{*} \in B^{*} \quad<x^{*}, f_{n}(.)>\rightarrow\|\|_{1}<x^{*}, f(.)>.
$$

Since $E^{\mathcal{A}_{n}}|L|<\infty$, there exists an $\mathcal{A}_{1}$-measurable partition $\left(B_{k}\right)_{k \geq 1}$ of $\Omega$ defined by

$$
B_{k}=\left\{\omega, k-1 \leq E^{\mathcal{A}_{1}}|L|(\omega)<k\right\} .
$$

For each $k \geq 1$, set $f_{n}^{k}=f_{n} 1_{B_{k}}$. So $\left(f_{n}^{k}, \mathcal{A}_{n}\right)_{n \geq 1}$ is a bounded pramart in $L_{E}^{1}(\mathcal{A})$.

Then from Akhiat and Ezzaki [3], there exists a unique martingale $\left(M_{n}^{k}\right)_{n \geq 1}$ in $L_{E}^{1}(\mathcal{A})$ and a pramart $\left(Z_{n}^{k}\right)_{n \geq 1}$ in $L_{E}^{1}(\mathcal{A})$ such that 


$$
f_{n}^{k}=M_{n}^{k}+Z_{n}^{k}, \forall n \geq 1,\left|Z_{n}^{k}\right| \rightarrow 0 \text { a.s. }
$$

Moreover for each $x^{*} \in E^{*}$,

$$
<x^{*}, f_{n}^{k}(.)>\rightarrow<x^{*}, 1_{B_{k}} f(.)>\text { a.s. }
$$

But as $<x^{*}, Z_{n}^{k}()>$. converge to Zero a.s.

We get

$$
\lim _{n}<x^{*}, M_{n}^{k}(.)>=<x^{*}, 1_{B_{k}} f(.)>\text { a.s. }
$$

Since $\left(M_{n}^{k}\right)_{n \geq 1}$ is a bounded martingale, so by Theorem 5.3.27 in Edgar [27].

$$
M_{n}^{k}(.) \rightarrow 1_{B_{k}} f(.) \text { a.s. } \forall k \geq 1
$$

Hence, the sequence $\left(f_{n}^{k}\right)_{n \geq 1}$ converge a.s. to $1_{B_{k}} f=f^{k} \forall k \geq 1$.

Set $M_{n}=\sum_{n \geq 1} M_{n}^{k} 1_{B_{k}}$

So,

$$
\lim _{n} M_{n}=\lim _{n} f_{n}=f \text { a.s. }
$$

Put.

$$
f^{k}(w)=\left\{\begin{array}{lr}
f(\omega), \text { if } & \omega \in B_{k}, \\
0, \text { if } & \omega \notin B_{k} .
\end{array}\right.
$$

Then there exists a negligible set $N=\cup_{k} N_{k}$, such that

$$
\forall \omega \in \Omega \backslash N, \lim _{n} f_{n}(\omega)=f(\omega) .
$$

Then by [16, Theorem 2.4], we have $\left\|f_{n}-f\right\|_{P e} \rightarrow 0$.

The second application of lemma 42 is the following equivalence between the convergence in Pettis norm of a Pettis integrable adapted sequence and the nature of his scalar product.

Lemma 44. Let L be a Pettis integrable random set with values in cwk $(E)$. Let $\left(f_{n}, \mathcal{A}_{n}\right)_{n \geq 1}$ be an adapted sequence in $P_{E}^{1}(\mathcal{A})$ such that $f_{n}(.) \in L(),. \forall n \geq 1$ and $E^{\mathcal{A}_{n}}|L|<\infty$ a.s. $\forall n \geq 1$.

Then

$$
f_{n} \rightarrow\|\|_{P_{e} f} \Leftrightarrow \forall x^{*} \in E^{*},\left(<x^{*}, f_{n}>\right)_{n \geq 1} \text { is a bounded pramart in } L_{\mathbb{R}}^{1} \text {. }
$$

Proof. If $f_{n} \rightarrow\|\|_{P e} f$, this implies that

$$
\lim _{n}<x^{*}, f_{n}>=<x^{*}, f>\text { in } L_{\mathbb{R}}^{1}, \forall x^{*} \in E^{*} .
$$

So

$$
\lim _{n}<x^{*}, f_{n}>=<x^{*}, f>\text { in probability, } \forall x^{*} \in E^{*} .
$$

On the other hand, since $f_{n}(.) \in L(),. \forall n \geq 1$, we have 


$$
\operatorname{supsup}_{n} \int_{x^{*} \in B^{*}} \int_{\Omega}\left|<x^{*}, f_{n}(\omega)>\right| d P \leq 2 \sup _{x^{*} \in B^{*}} \int_{\Omega}\left|\delta^{*}\left(x^{*}, L(\omega)\right)\right| d P<\infty .
$$

So, by [28, Lemma 6] $\forall x^{*} \in E^{*}\left(<x^{*}, f_{n}(.)>, \mathcal{A}_{n}\right)_{n \geq 1}$ is a bounded pramart in $L_{\mathbb{R}}^{1}$.

The converse is a direct consequence of Lemma 43 .

By using theorem 36 and a direct application of lemma 42, we prove the convergence in weak topology of $c w k(E)$-valued Pettis integrable pramart.

Lemma 45. Let $\left(X_{n}\right)_{n \geq 1}$ be a sequence of Pettis integrable pramart with values in cwk $(E)$ satisfying the following conditions:

(1) There exists a Pettis integrable random set $L$ with values in $c w k(E)$ such that $X_{n} \subset L$ $\forall n \geq 1$,

(2) For each $n \geq 1, E^{\mathcal{A}_{n}}|L|<\infty$.

Then there exists a Pettis integrable random set $X$ with values in cwk(E) such that

$$
\lim _{n} \delta^{*}\left(x^{*}, X_{n}(\omega)\right)=\delta^{*}\left(x^{*}, X(\omega)\right), \text { a.s. } \forall x^{*} \in E^{*} .
$$

Proof. By theorem 36, $E^{\mathcal{A}_{\sigma}} X_{\tau}$ exists $\forall \sigma, \tau \in T$ and $\tau \geq \sigma$. Then the notion of Pettis integrable pramart with values in $c w k(E)$ is well defined.

Since $\left(X_{n}\right)_{n \geq 1}$ is a pramart in $P_{c w k(E)}^{1}(\mathcal{A})$ then $\left(\delta^{*}\left(x^{*}, X_{n}\right)\right)_{n \geq 1}$ is a pramart too. By hypothesis $X_{n}(\omega) \subset L(\omega)$ for all $n \in \mathbb{N}^{*}$ and for all $\omega \in \Omega$ then

$$
\sup _{n} \sup _{x^{*} \in B^{*}} \int_{\Omega}\left|\delta^{*}\left(x^{*}, X_{n}(\omega)\right)\right| d P \leq 2 \sup _{x^{*} \in B^{*}} \int_{\Omega}\left|\delta^{*}\left(x^{*}, L(\omega)\right)\right| d P<\infty .
$$

So $\left(\delta^{*}\left(x^{*}, X_{n}\right), \mathcal{A}_{n}\right)_{n \geq 1}$ is a $L^{1}$-bounded pramart, we deduce that it admits a limit.

Then by lemma 42 , there exists a Pettis integrable and measurable random set $X$ with values in $\operatorname{cwk}(E)$ such that

$$
\lim _{n} \delta^{*}\left(x^{*}, X_{n}\right)=\delta^{*}\left(x^{*}, X\right), \text { a.s. } \forall x^{*} \in E^{*} .
$$

The following theorem is a new version in Pettis integration of theorem 3.3 in Choukairi [5] provided in Bochner integration.

Theorem 46. Let $\left(X_{n}\right)_{n>1}$ be a Pettis integrable pramart with values in cwk(E) that satisfying the following conditions:

(1) There exists a Pettis integrable random set $L$ with values in $c w k(E)$ such that $X_{n} \subset L$ $\forall n \geq 1$,

(2) For each $n \geq 1, E^{\mathcal{A}_{n}}|L|<\infty$.

Then there exists a Pettis integrable random set $X$ with values in cwk $(E)$ such that.

(a) $\lim _{n \rightarrow \infty} \delta^{*}\left(x^{*}, \int_{A} X_{n} d P\right)=\delta^{*}\left(x^{*}, \int_{A} X d P\right), \quad \forall x^{*} \in E^{*}$ and for every $A \in \mathcal{A}$.

(b) $M-\lim _{n \rightarrow \infty} E^{\mathcal{A}_{n}} X=X$ a.s.

(c) $s-l i S_{X_{n}}^{P e} \subset S_{X}^{P e}$.

Proof. From lemma 45, there exists a Pettis integrable and measurable random set $X$ with values in $c w k(E)$ such that

$$
\lim _{n} \delta^{*}\left(x^{*}, X_{n}\right)=\delta^{*}\left(x^{*}, X\right), \text { a.s. } \forall x^{*} \in E^{*} .
$$


By uniform integrability of $\left\{\delta^{*}\left(x^{*}, L\right), x^{*} \in B^{*}\right\}$ we conclude that $\left\{\delta^{*}\left(x^{*}, X_{n}\right), x^{*} \in B^{*}, n \geq 1\right\}$ is uniformly integrable.

Then by (46.1) and the uniform integrability of $\left\{\delta^{*}\left(x^{*}, X_{n}\right), n \geq 1\right\}$ we have for $x^{*} \in E^{*}$ and for every $A \in \mathcal{A}$,

$$
\begin{aligned}
\lim _{n \rightarrow \infty} \delta^{*}\left(x^{*}, \int_{A} X_{n} d P\right) & =\lim _{n \rightarrow \infty} \int_{A} \delta^{*}\left(x^{*}, X_{n}\right) d P \\
& =\int_{A} \delta^{*}\left(x^{*}, X\right) d P=\delta^{*}\left(x^{*}, \int_{A} X d P\right)
\end{aligned}
$$

(b) $M-\lim _{n \rightarrow \infty} E^{\mathcal{A}_{n}} X=X$ a.s.

By (2) and theorem $34, E^{\mathcal{A}_{n}} X$ exists for each $n \geq 1$ by El Allali et Ezzaki[12, Theorem 4.11], we have

$$
M-\lim _{n \rightarrow \infty} E^{\mathcal{A}_{n}} X(.)=X(.) \text { a.s. }
$$

(c) Let $f \in s-l i S_{X_{n}}^{P e}$ by definition of $s-l i$, there exists $\left(f_{n}\right)_{n \geq 1} \in\left(S_{X_{n}}^{P e}\right)_{n \geq 1}$ such that

$$
f_{n} \rightarrow\|\|_{P e} f .
$$

Then by lemma $44,\left(<x^{*}, f_{n}()>., \mathcal{A}_{n}\right)$ is a bounded pramart in $L_{\mathbb{R}}^{1}$, and

$$
<x^{*}, f>=\lim _{n}<x^{*}, f_{n}>\text { in } L_{\mathbb{R}}^{1}, \forall x^{*} \in E^{*} .
$$

$\operatorname{By}(a), \int_{A} f d P \in \int_{A} X d P, \forall A \in \mathcal{A}$,

and

$$
<x_{m}^{*}, f>\leq \delta^{*}\left(x_{m}^{*}, X\right) \text { a.s., for each } x_{m}^{*} \in D^{*} .
$$

Then there exists a negligible set $N=\cup_{m} N_{x_{m}}$ such that $\forall \omega \in \Omega \backslash N, f(\omega) \in X(\omega)$.

Since $X$ is in $P_{c w k(E)}^{1}$, hence $f$ is Pettis integrable. From (46.2) and proposition $311 f \in S_{X}^{P e}$.

The following lemma is a generalization in Pettis integration of lemma 6.1 in Castaing et al. [29].

Lemma 47. Assume that $\left(x_{m}^{*}\right)_{m \geq 1}$ is a dense sequence in $B^{*}$ with respect to the Mackey topology. Let $X \in P_{\operatorname{cwk}(E)}^{1}(\mathcal{A})$ such that $E^{\mathcal{A}_{1}}|X|<\infty$.

Let $g$ be a positive random variable such that $E^{\mathcal{A}_{1}} g<\infty$, and $\left(X_{n}\right)_{n \geq 1}$ be a pramart in $P_{\text {cwk(E) }}^{1}(\mathcal{A})$ such that $\left|X_{n}\right| \leq g$.

Then the following holds:

$$
\begin{aligned}
& {\left[\delta^{*}\left(x_{m}^{*}, E^{\mathcal{A}_{\sigma}} X(.)\right)-\delta^{*}\left(x_{m}^{*}, X_{\sigma}(.)\right)\right]^{+}-E^{\mathcal{A}_{\sigma}} \delta^{*}\left(x_{m}^{*}, E^{\mathcal{A}_{\tau}} X(.)\right)} \\
& \quad-\delta^{*}\left(x_{m}^{*}, X_{\tau}(.)\right)^{+} \leq\left|\delta^{*}\left(x_{m}^{*}, E^{\mathcal{A}_{\sigma}} X_{\tau}(.)\right)-\delta^{*}\left(x_{m}^{*}, X_{\sigma}(.)\right)\right| \leq H\left(E^{\mathcal{A}_{\sigma}} X_{\tau}(.), X_{\sigma}(.)\right) \text { a.s. }
\end{aligned}
$$

for all $m \geq 1, \sigma, \tau \in T, \tau \geq \sigma$. 
Proof. From theorem $36, E^{\mathcal{A}_{\sigma}} X_{\tau}$ exists $\forall \tau \geq \sigma$ and $E^{\mathcal{A}_{\sigma}} X$ exists. We may apply the techniques developed by Castaing et al. [29]. For each $m, n \geq 1$, let us set

$$
\varphi_{m, n}(.)=\delta^{*}\left(x_{m}^{*}, E^{\mathcal{A}_{n}} X(.)\right)-\delta^{*}\left(x_{m}^{*}, X_{n}(.)\right) .
$$

Let $\sigma, \tau \in T, \tau \geq \sigma$ and let

$$
\begin{aligned}
& \varphi_{m, \tau}(.)=\delta^{*}\left(x_{m}^{*}, E^{\mathcal{A}_{\tau}} X(.)\right)-\delta^{*}\left(x_{m}^{*}, X_{\tau}(.)\right), \\
& \varphi_{m, \sigma}(.)=\delta^{*}\left(x_{m}^{*}, E^{\mathcal{A}_{\sigma}} X(.)\right)-\delta^{*}\left(x_{m}^{*}, X_{\sigma}(.)\right) .
\end{aligned}
$$

Now we prove that $\left|E^{\mathcal{A}_{\sigma}} \varphi_{m, \tau}().\right| \leq E^{\mathcal{A}_{\sigma}}\left|\varphi_{m, \tau}().\right|$ a.s.

Let $\left(x_{m}^{*}\right)_{m \geq 1}$ be a dense sequence in $B^{*}$. Since $\left(X_{n}, \mathcal{A}_{n}\right)_{n \geq 1}$ is a sequence of Pettis integrable pramart with values in $c w k(E)$, then for each $x_{m}^{*} \in B^{*}$ we have $\left(\delta^{*}\left(x_{m}^{*}, X_{n}().\right)\right)$ is a real valued pramart.

For each $x_{m}^{*} \in B^{*}$ by theorem 36 ,

$$
\begin{aligned}
\int_{A} E^{\mathcal{A}_{\sigma}} \delta^{*}\left(x_{m}^{*}, X_{\tau}(.)\right) d P & =\int_{A} \delta^{*}\left(x_{m}^{*}, X_{\tau}(.)\right) d P \\
& =\delta^{*}\left(x_{m}^{*}, \int_{A} X_{\tau}(.) d P\right) \\
& =\delta^{*}\left(x_{m}^{*}, \int_{A} E^{\mathcal{A}_{\sigma}} X_{\tau}(.) d P\right)=\int_{A} \delta^{*}\left(x_{m}^{*}, E^{\mathcal{A}_{\sigma}} X_{\tau}(.)\right) d P .
\end{aligned}
$$

Then

$$
E^{\mathcal{A}_{\sigma}} \delta^{*}\left(x_{m}^{*}, X_{\tau}(.)\right)=\delta^{*}\left(x_{m}^{*}, E^{\mathcal{A}_{\sigma}} X_{\tau}(.)\right) \text { a.s. }
$$

Thus by applying the Jensen's inequality for every $m \geq 1\left|E^{\mathcal{A}_{\sigma}} \varphi_{m, \tau}().\right| \leq E^{\mathcal{A}_{\sigma}}\left|\varphi_{m, \tau}().\right|$ a.s.

Then we have,

$$
\begin{aligned}
\boldsymbol{\varphi}_{m, \sigma}(.)^{+}-E^{\mathcal{A}_{\sigma}} \boldsymbol{\varphi}_{m, \tau}(.)^{+} & =\frac{1}{2}\left[\boldsymbol{\varphi}_{m, \sigma}(.)+\left|\boldsymbol{\varphi}_{m, \sigma}(.)\right|-E^{\mathcal{A}_{\sigma}} \boldsymbol{\varphi}_{m, \tau}(.)-E^{\mathcal{A}_{\sigma}}\left(\left|\varphi_{m, \tau}(.)\right|\right)\right] \\
& =\frac{1}{2}\left[\boldsymbol{\varphi}_{m, \sigma}(.)-E^{\mathcal{A}_{\sigma}} \boldsymbol{\varphi}_{m, \tau}(.)+\left|\boldsymbol{\varphi}_{m, \sigma}(.)\right|-E^{\mathcal{A}_{\sigma}}\left|\boldsymbol{\varphi}_{m, \tau}(.)\right|\right] \\
& \leq \frac{1}{2}\left[\boldsymbol{\varphi}_{m, \sigma}(.)-E^{\mathcal{A}_{\sigma}} \boldsymbol{\varphi}_{m, \tau}(.)+\left|\boldsymbol{\varphi}_{m, \sigma}(.)-E^{\mathcal{A}_{\sigma}} \boldsymbol{\varphi}_{m, \tau}(.)\right|\right] \\
& =\left[\boldsymbol{\varphi}_{m, \sigma}(.)-E^{\mathcal{A}_{\sigma}} \boldsymbol{\varphi}_{m, \tau}(.)\right]^{+} \\
& =\left[\delta^{*}\left(x_{m}^{*}, E^{\mathcal{A}_{\sigma}} X_{\tau}(.)\right)-\delta^{*}\left(x_{m}^{*}, X_{\sigma}(.)\right)\right]^{+} \\
& \leq H\left(E^{\mathcal{A}_{\sigma}} X_{\tau}(.), X_{\sigma}(.)\right) \text { a.s. }
\end{aligned}
$$


Consequently, by definition VIII.1.14 of Egghe [6], $\left(\left(\left[\delta^{*}\left(x_{m}^{*}, E^{\mathcal{A}_{n}} X\right)-\delta^{*}\left(x_{m}^{*}, X_{n}\right)\right]^{+}\right)_{n \geq 1}\right)_{m \geq 1}$ is uniform sequence of subpramarts.

Now, we present some convergence results of set valued Pettis integrable pramarts with convex weakly compact values in a separable Banach space $E$.

Theorem 48. Let $\left(X_{n}\right)_{n \geq 1}$ be a Pettis integrable pramart with values in cwk $(E)$ satisfying the following conditions:

(1) There exists a Pettis integrable random set $L$ with values in cwk $(E)$ such that $X_{n} \subset L \forall n \geq 1$.

(2) For each $n \geq 1, E^{\mathcal{A}_{n}}|L|<\infty$.

Then there exists a Pettis integrable random set $X$ with values in cwk $(E)$ such that.

(a) $\lim _{n \rightarrow \infty} H\left(E^{\mathcal{A}_{n}} X, X_{n}\right)=0$ a.s.

(b) $M-\lim _{n \rightarrow \infty} X_{n}=X$ a.s.

(c) $\lim _{n \rightarrow \infty} d\left(x, X_{n}\right)=d(x, X)$ a.s. $\forall x \in E$.

Proof. (a) We will prove that $\lim _{n \rightarrow \infty} H\left(E^{\mathcal{A}_{n}} X, X_{n}\right)=0$ a.s.

By theorem 46, we have

$$
M-\lim _{n \rightarrow \infty} E^{\mathcal{A}_{n}} X=X \text { a.s. }
$$

From lemma 45,

$$
\lim _{n \rightarrow \infty} \delta^{*}\left(x^{*}, X_{n}\right)=\delta^{*}\left(x^{*}, X\right) \text { a.s. } \forall x^{*} \in E^{*} .
$$

By (48.1) there exists a negligible $N$ such that $\forall \omega \in \Omega \backslash N, \forall x^{*} \in E^{*}$

$$
\delta^{*}\left(x^{*}, X(\omega)\right)=\lim _{n \rightarrow \infty} \delta^{*}\left(x^{*}, X_{n}(\omega)\right) \leq \delta^{*}\left(x^{*}, L(\omega)\right)
$$

Then $X(\omega) \subset L(\omega)$ so, $E^{\mathcal{A}_{n}}|X().| \leq E^{\mathcal{A}_{n}}|L()$.$| a.s. \forall n \geq 1$.

From (1) and (2) $E^{\mathcal{A}_{1}}\left|X_{n}\right| \leq E^{\mathcal{A}_{1}}|L|<\infty$ and $\left(X_{n}, \mathcal{A}_{n}\right)_{n \geq 1}$ is a pramart.

Let $\left(x_{k}^{*}\right)_{k \geq 1}$ be a dense sequence in $B^{*}$ with respect to the Mackey topology. By lemma 47 the sequence $\left(\left(\delta^{*}\left(x_{k}^{*}, E^{\mathcal{A}_{n}} X\right)-\delta^{*}\left(x_{k}^{*}, X_{n}\right)\right)_{n \geq 1}\right)_{k \geq 1}$ is uniform subpramart.

By (2) there exists a partition $\left(A_{j}\right)_{j \geq 1}$ of $\Omega$ in $\mathcal{A}_{1}$ defined by

$$
A_{j}=\left\{j-1 \leq E^{\mathcal{A}_{1}}|L|<j\right\} .
$$

Since $\left|X_{n}\right| \leq|L|$, then

$$
H\left(E^{\mathcal{A}_{n}} X, X_{n}\right) \leq E^{\mathcal{A}_{n}}|L|+|L| \text { a.s. }
$$

So,

$$
\int_{A_{j}} H\left(E^{\mathcal{A}_{n}} X, X_{n}\right) d P \leq \int_{A_{j}}\left(E^{\mathcal{A}_{n}}|L|+|L|\right) d P .
$$


For each $n \geq 1$,

Pettis

$$
\int_{A_{j}} H\left(E^{\mathcal{A}_{n}} X, X_{n}\right) d P \leq \int_{A_{j}} E^{\mathcal{A}_{n}}|L| d P+\int_{A_{j}}|L| d P .
$$

integrable multivalued pramart

Since $A_{j} \in \mathcal{A}_{1}$ then $A_{j} \in \mathcal{A}_{n}$.

So, by definition of the conditional expectation of the positive random variable

$$
\int_{A_{j}} E^{\mathcal{A}_{n}}|L| d P=\int_{A_{j}}|L| d P
$$

By (48.2) and (48.3),

$$
\int_{A_{j}} H\left(E^{\mathcal{A}_{n}} X, X_{n}\right) d P \leq 2 \int_{A_{j}}|L| d P=2 \int_{A_{j}} E^{\mathcal{A}_{1}}|L| d P \leq 2 j P\left(A_{j}\right)<\infty .
$$

Then

$$
\sup _{n \geq 1} \int_{A_{j}} H\left(E^{\mathcal{A}_{n}} X, X_{n}\right) d P<\infty .
$$

So for every $j \geq 1$,

$$
\sup _{n \geq 1} \int_{A_{j}} \sup _{k}\left(\delta^{*}\left(x_{k}^{*}, E^{\mathcal{A}_{n}} X\right)-\delta^{*}\left(x_{k}^{*}, X_{n}\right)\right)^{+} d P \leq \sup _{n \geq 1} \int_{A_{j}} H\left(E^{\mathcal{A}_{n}} X, X_{n}\right) d P<\infty .
$$

Then $\left(\left(\left[\delta^{*}\left(x_{k}^{*}, E^{\mathcal{A}_{n}} X\right)-\delta^{*}\left(x_{k}^{*}, X_{n}\right)\right]^{+}\right)_{n \geq 1}\right)_{k \geq 1}$ is a bounded uniform sequence of positive subpramarts on $A_{j}$. Applying Egghe [6, Lemma VIII.1.15], there exists a negligible $N_{j}^{k}$ such that $\forall \omega \in A_{j} \backslash N_{j}^{k}$

$$
\begin{aligned}
\limsup _{n \rightarrow \infty}\left(\delta^{*}\left(x_{k}^{*}, E^{\mathcal{A}_{n}} X(\omega)\right)-\delta^{*}\left(x_{k}^{*}, X_{n}(\omega)\right)\right) & =\sup _{k} \lim _{n \rightarrow \infty}\left(\delta^{*}\left(x_{k}^{*}, E^{\mathcal{A}_{n}} X(\omega)\right)-\delta^{*}\left(x_{k}^{*}, X_{n}(\omega)\right)\right) \\
& =0 .
\end{aligned}
$$

Since $\left(A_{j}\right)_{j \geq 1}$ is a partition of $\Omega$ then there exists a negligible $N=\cup_{j \geq 1} \cup_{k \geq 1} N_{j}^{k}$ such that $\forall \omega \in \Omega \mid \mathrm{N}$

$$
\lim _{n \rightarrow \infty} H\left(E^{\mathcal{A}_{n}} X(\omega), X_{n}(\omega)\right)=0 .
$$

(b) Since $\lim _{n \rightarrow \infty} H\left(E^{\mathcal{A}_{n}} X, X_{n}\right)=0$ a.s. so, (b) is deduced from proposition 27.

(c) Now we will prove that $\lim _{n} d\left(x, X_{n}().\right)=d(x, X()),. a . s . \forall x \in E$.

From the scalarly convergence of $\left(X_{n}\right)_{n \geq 1}$ and proposition 28 we get

$$
\lim _{n} d\left(x, X_{n}(.)\right)=d(x, X(.)) \text {, a.s. } \forall x \in E \text {. }
$$

When the pramarts $\left(X_{n}\right)_{n \geq 1}$ in theorem 48 are single-valued, we have the following corollary.

Corollary 49. Let $\left(X_{n}, \mathcal{A}_{n}\right)_{n \geq 1}$ be a sequence of Pettis integrable E-valued pramart such that:

(1) There exists a Pettis integrable random set $L$ with values in $c w k(E)$ such that $X_{n}(\omega) \in L(\omega) \forall n \geq 1$ and $\omega \in \Omega$,

(2) For each $n \geq 1, E^{\mathcal{A}_{n}}|L|<\infty$.

Then there exists a Pettis integrable random variable $X$ with values in $E$ such that 
AJMS

$$
\lim _{n \rightarrow \infty}\left\|E^{\mathcal{A}_{n}} X-X_{n}\right\|=0 \text { a.s. }
$$

So that

$$
\lim _{n \rightarrow \infty}\left\|X_{n}-X\right\|=0 \text { a.s. }
$$

Proof. Since $\left(X_{n}, \mathcal{A}_{n}\right)_{n \geq 1}$ is a Pettis integrable pramart and $X_{n}(\omega) \in L(\omega)$ for each $n \geq 1$ and each $\omega \in \Omega$ then

$$
\sup _{n} \sup _{x^{*} \in B^{*}} \int_{\Omega}\left|<x^{*}, X_{n}(\omega)>\right| d P \leq 2 \sup _{x^{*} \in B^{*}} \int_{\Omega}\left|\delta^{*}\left(x^{*}, L(\omega)\right)\right| d P<\infty .
$$

So, the $L^{1}$-bounded pramart $\left(<x^{*}, X_{n}(.)>\right)_{n \geq 1}$ converges a.s. Then $\lim _{n}<x^{*}, X_{n}()>$. exists.

Since $L$ is in $P_{\operatorname{cok}(E)}^{1}$ by lemma 42 , there exists $X \in P_{E}^{1}$ such that

$$
\lim _{n \rightarrow \infty}<x^{*}, X_{n}>=<x^{*}, X>\text { a.s., } \forall x^{*} \in E^{*} .
$$

By (2) and theorem $31, E^{\mathcal{A}_{n}} X$ exists. So consider the regular martingale $E^{\mathcal{A}_{n}} X$ such that

$$
X_{n}=E^{\mathcal{A}_{n}} X+\left(X_{n}-E^{\mathcal{A}_{n}} X\right) .
$$

By theorem 48 , we have

$$
\lim _{n \rightarrow \infty}\left\|E^{\mathcal{A}_{n}} X-X_{n}\right\|=0 \text { a.s. }
$$

By applying Akhiat, Castaing et Ezzaki [1, Proposition 5.1] we have $\lim _{n \rightarrow \infty} E^{\mathcal{A}_{n}} X=X$ a.s.

Then we conclude that

$$
\lim _{n \rightarrow \infty}\left\|X_{n}-X\right\|=0 \text { a.s. }
$$

The following theorem stated in Pettis integration generalizes a result obtained in Castaing and Salvadori [4, theorem 6.9] in Bochner integration.

Theorem 410. Assume that E has RNP and $E^{*}$ has RNP.

Let $\left(X_{n}\right)_{n \geq 1}$ be a Pettis integrable pramart with values in $P_{c w k(E)}^{1}(\mathcal{A})$ satisfying the following conditions:

(1) $\left|X_{n}\right| \leq g$ for all $n \geq 1$ where $g$ is a positive function such that $E^{\mathcal{A}_{1}} g<\infty$,

(2) $\left\{\delta^{*}\left(x^{*}, X_{n}\right), n \geq 1\right\}$ is uniformly integrable,

(3) For each $A \in \mathcal{A}$, the set $\left\{\cup_{n} \geq 1 \int_{A} X_{n} d P, n \geq 1\right\}$ is relatively weakly compact. Then there exists a Pettis integrable random set $X$ with values in $c w k(E)$ such that. 
(a) $\lim _{n \rightarrow \infty} H\left(E^{\mathcal{A}_{n}} X, X_{n}\right)=0$ a.s.

Pettis

(b) $M-\lim _{n \rightarrow \infty} X_{n}=X$ a.s.

integrable

(c) $\lim _{n \rightarrow \infty} d\left(x, X_{n}\right)=d(x, X)$ a.s. $\forall x \in E$.

multivalued

pramart

Proof. We will prove that

$$
\lim _{n \rightarrow \infty} \delta^{*}\left(x^{*}, X_{n}(.)\right)=\delta^{*}\left(x^{*}, X(.)\right), \text { a.s. } \forall x^{*} \in E^{*} .
$$

Further, from (2) for each $x^{*} \in E^{*}$, the $L^{1}$-bounded pramart $\left(\delta^{*}\left(x^{*}, X_{n}\right)\right)_{n \geq 1}$ converge a.s.

In other words for each $x^{*} \in E^{*}$ there exists a function $\varphi_{x^{*}}$ such that

$$
\lim _{n} \delta^{*}\left(x^{*}, X_{n}\right)=\varphi_{x^{*}} \text { a.s. }
$$

Now assume that for each $A \in \mathcal{A}$, the set $\left\{\int_{A} X_{n} d P, n \geq 1\right\}$ is include in a convex weakly compact subset denoted by $K_{A}$.

Set $\forall n \geq 1 M_{n}(A)=\int_{A} X_{n} d P$, for each $A \in \mathcal{A}$.

Since $\left(X_{n}, \mathcal{A}_{n}\right)_{n \geq 1}$ is with values in $c w k(E)$. Then by El Amri and Hess [15, Theorem 5.4] $M_{n}($.$) is a c w k(E)$-valued.

The fact that, $\left(X_{n}, \mathcal{A}_{n}\right)_{n \geq 1}$ is Pettis integrable, implies that $M_{n}$ is a multimeasure for all $n \geq 1$.

$$
\delta^{*}\left(x^{*}, M_{n}(A)\right)=\delta^{*}\left(x^{*}, \int_{A} X_{n} d P\right)=\int_{A} \delta^{*}\left(x^{*}, X_{n}\right) d P .
$$

$\forall x^{*} \in E^{*}$ set,

$$
\psi_{A}\left(x^{*}\right)=\int_{A} \lim _{n} \delta^{*}\left(x^{*}, X_{n}\right) d P
$$

By (2) and (410.1) a sequence $\left(\delta^{*}\left(x^{*}, X_{n}(.)\right)_{n \geq 1}\right.$ converges in $L^{1}$ to $\varphi_{x^{*}} \forall x^{*} \in E^{*}$.

Then $\forall A \in \mathcal{A}$,

$$
\lim _{n} \int_{A} \delta^{*}\left(x^{*}, X_{n}\right) d P=\int_{A} \lim _{n} \delta^{*}\left(x^{*}, X_{n}\right) d P=\int_{A} \varphi_{x^{*}} d P
$$

So,

$$
\psi_{A}\left(x^{*}\right)=\lim _{n} \delta^{*}\left(x^{*}, \int_{A} X_{n} d P\right) \leq \delta^{*}\left(x^{*}, K_{A}\right) .
$$

Since for each. $A \in \mathcal{A} M_{n}(A)=\int_{A} X_{n} d P$

So,

$$
\lim _{n} \delta^{*}\left(x^{*}, M_{n}(A)\right)=\psi_{A}\left(x^{*}\right) \leq \delta^{*}\left(x^{*}, K_{A}\right) \forall x^{*} \in E^{*} .
$$


By lemma 42 there exists $M(A) \in P_{\operatorname{cwk}(E)}^{1}(\mathcal{A})$ such that,

$$
\lim _{n} \delta^{*}\left(x^{*}, M_{n}(A)\right)=\delta^{*}\left(x^{*}, M(A)\right)=\psi_{A}\left(x^{*}\right), \text { for all } x^{*} \in E^{*}, A \in \mathcal{A} .
$$

By (410.2)

$$
\delta^{*}\left(x^{*}, M(A)\right)=\int_{A} \varphi_{x^{*}} d P
$$

Since $\varphi_{x^{*}} \in L^{1}$ then for every $x^{*} \in E^{*}, \delta^{*}\left(x^{*}, M().\right)$ is a finite scalar measure. So $M$ is a multimeasure.

Let proof that $M$ is of $\sigma$-bounded variation.

As $E^{\mathcal{A}_{1}} g<\infty$, then there exists $\left(B_{k}\right)_{k \geq 1}$ a partition of $\Omega$ in $\mathcal{A}_{1}$ such that

$$
\int_{B_{k}} g d P<\infty, \forall k \geq 1
$$

Let $B_{j}$ fixed and $\left(A_{i}\right)_{i \geq 1}$ a partition of $B_{j}$ then

$$
\begin{aligned}
\left|M\left(A_{i} \cap B_{j}\right)\right| & =\sup _{x^{*} \in B^{*} n} \lim _{n} \delta^{*}\left(x^{*}, M_{n}\left(A_{i} \cap B_{j}\right)\right) \\
& \leq \lim _{n} \sup _{x^{*} \in B^{*}} \delta^{*}\left(x^{*}, M_{n}\left(A_{i} \cap B_{j}\right)\right) \\
& \leq \sup _{n} \int_{A_{i} \cap B_{j}}\left|X_{n}\right| d P \leq \int_{A_{i} \cap B_{j}} g d P \\
& \leq \int_{B_{j}} g d P<\infty .
\end{aligned}
$$

Then

$$
\sum_{i=1}^{n}\left|M\left(A_{i} \cap B_{j}\right)\right| \leq \sum_{i=1}^{n} \int_{A_{i} \cap B_{j}} g d P=\int_{B_{j}} g d P .
$$

So, $M$ is of $\sigma$-bounded variation then by theorem 4.8 in Ziat [19] there exists $\mathcal{A}$-measurable multifunction $X$ in $P_{c w k(E)}^{1}(\mathcal{A})$ such that

$$
M(A)=\int_{A} X d P \forall A \in \mathcal{A} .
$$

Then

Hence

$$
\begin{aligned}
\lim _{n} \delta^{*}\left(x^{*}, \int_{A} X_{n} d P\right) & =\delta^{*}\left(x^{*}, \int_{A} X d P\right) \\
& =\int_{A} \delta^{*}\left(x^{*}, X\right) d P \\
& =\int_{A} \lim \delta^{*}\left(x^{*}, X_{n}\right) d P .
\end{aligned}
$$

$$
\delta^{*}\left(x^{*}, X\right)=\lim _{n} \delta^{*}\left(x^{*}, X_{n}\right) \text { a.s., } \forall x^{*} \in E^{*} .
$$

If we put $|L|=g$, so (a), (b) and (c) follow as in the proof of theorem 48 . 


\section{References}

1. Akhiat F, Castaing C, Ezzaki, F. Some various convergence results for multivalued martingales. Advances in mathematical economics; Tokyo: Springer; 2010; 13: 1-33.

2. Akhiat F, El Harami M, Ezzaki F. Pettis conditional expectation of closed convex random sets in a Banach space without RNP. J. Korean Math. Soc. 2018; 55(4): 833-48.

3. Akhiat F, Ezzaki F. Representation theorems for multivalued pramarts. J Korean Math Soc. 2013; 50(1): $1-16$.

4. Castaing C, Salvadori A. Convergence results for a class of pramarts and superpramarts in Banach spaces. Commentationes Mathematicae. 2013; 53(2): 223-53.

5. Choukairi-Dini A. On almost sure convergence of vector valued pramarts and multivalued pramarts. J Convex Anal. 1996; 3: 245-54.

6. Egghe L. Stopping time techniques for analysts and probabilists. London Mathematical Society Lecture Note. Cambridge University Press; 1984: 100.

7. El-louh M, Ezzaki F. Some properties of pettis integrable multivalued martingales. Proceedings of the Mediterranean Symposium on Smart City Applications. Cham. Springer: 2017: 1028-34.

8. Ezzaki F, Tahri K. Representation theorem of set valued regular martingale: application to the convergence of set valued martingale. Stat Probab Lett. 2019; 154: 108548.

9. Talagrand M. Some structure results for martingales in the limit and pramarts. The Ann Probab. 1985; 13(4): 1192-203.

10. Musial K. Topics in the theory of Pettis integration. Rend Istit Mat Univ Trieste. 1991; 23(1): 177-262.

11. Talagrand M. Pettis integral and measure theory. Mem American Mathematical Soc. Rhode Island. 1984: 51.

12. El Allali M, Ezzaki F. Conditional expectation of Pettis integrable random sets: existence and convergence theorems. Adv Oper Theor. 2021; 6(1): 1-19.

13. Castaing C, Valadier M. Convex analysis and measurable multifunctions. Lecture notes in mathematics. Berlin, Heidelberg: Springer. 1977: 580.

14. Beer G. Support and distance functional for convex sets. Numer Funct Anal Optim. 1989; 10(12): 15-36.

15. El Amri K, Hess C. On the pettis integral of closed valued multifunctions. Set-Valued Anal. 2000; 8(4): 329-60.

16. Balcerzak M, Musial K. Vitali type convergence theorems for Banach space valued integrals. Acta Math Sinica, English Ser. 2013; 29(11): 2027-36.

17. El Harami M, Ezzaki F. General Pettis conditional expectation and convergence theorems. Int J Mathematics Stat. 2012; 11(1): 91-111.

18. El-louh M, Ezzaki F, Tahri K. Set valued Aumann-Pettis integrable martingale representation theorem and convergence. Ann Funct Anal. 2020; 11(4): 1236-56.

19. Ziat H. Approximation and Decomposability in the space of Pettis integrable functions. Int J Math Anal. 2012; 6(31): 1519-37.

20. El Allali M, El-louh M, Ezzaki F. On the convergence and regularity of Aumann-Pettis integrable multivalued martingales. J Convex Anal. 2020; 27(4).

21. Barcenas D, Urbina W. Measurable multifunctions in non separable Banach spaces. SIAM J Math Anal Sep. 1997; 28(5): 1212-26.

22. Hiai F, Umegaki H. Integrals, conditional expectations, and martingales of multivalued functions. J Multivariate Anal. 1977; 7(1): 149-82.

23. Costé A. Contribution à la théorie de l'intégration multivoque. Paris: Thèse d'Etat, Université Pierre et Marie Curie; 1977. 
24. Hess C. On multivalued martingales whose values may be unbounded: martingale selectors and Mosco convergence. J Multivar Anal. 1991; 39(1): 175-201.

25. Uhl JJ. Martingales of strongly measurable Pettis integrable functions. Trans Am Math Soc. 1972; 167: 369-78.

26. Egghe L. Strong convergence of pramarts in Banach spaces. Can J Math. 1981; 33(2): 357-61.

27. Edgar GA, Sucheston L, Edgar G. Stopping times and directed processes (No. 47). Cambridge: Cambridge University Press; 1992.

28. Wang ZP, Xue XH. Some remarks on pramarts and mils. Glasg Math J. 1993; 35(2): 239-51.

29. Castaing C, Ezzaki F, Tahri K. Convergences of multivalued pramarts. J. Nonlinear Convex Anal. 2010; 11(2): 243-66.

\section{Corresponding author}

M'Hamed El-Louh can be contacted at: ellouh.mhamed@gmail.com

For instructions on how to order reprints of this article, please visit our website: 